\title{
Article \\ Arabidopsis G-Protein $\beta$ Subunit AGB1 Negatively Regulates DNA Binding of MYB62, a Suppressor in the Gibberellin Pathway
}

\author{
Xin $\mathrm{Qi}^{1,2,+}$, Wensi Tang ${ }^{1,+}{ }^{1}$, Weiwei $\mathrm{Li}^{3}$, Zhang He ${ }^{1}$, Weiya Xu ${ }^{1}$, Zhijin Fan $^{2}{ }^{\oplus}$, Yongbin Zhou ${ }^{1}$, \\ Chunxiao Wang ${ }^{1}$, Zhaoshi $\mathrm{Xu}^{1}{ }^{1}{ }^{\text {, Jun Chen }}{ }^{1}$, Shiqin Gao ${ }^{4}$, Youzhi Ma ${ }^{1, *}$ and Ming Chen ${ }^{1, *}$ \\ 1 Institute of Crop Sciences, Chinese Academy of Agricultural Sciences (CAAS)/National Key Facility for Crop \\ Gene Resources and Genetic Improvement, Key Laboratory of Biology and Genetic Improvement of Triticeae \\ Crops, Ministry of Agriculture, Beijing 100081, China; 13121253699@163.com (X.Q.); \\ tang_wensi@yeah.net (W.T.); hz384298890@gmail.com (Z.H.); 15651912225@163.com (W.X.); \\ zhouyongbin@caas.cn (Y.Z.); wcx51619@163.com (C.W.); xuzhaoshi@caas.cn (Z.X.); chenjun01@caas.cn (J.C.) \\ 2 State Key Laboratory of Elemento-Organic Chemistry, College of Chemistry, Nankai University, \\ Tianjin 300071, China; fanzj@nankai.edu.cn \\ 3 Beijing Advanced Innovation Center for Food Nutrition and Human Health, Beijing Technology \& Business \\ University (BTBU), Beijing 100048, China; liweiwei.0304@163.com \\ 4 Beijing Engineering Research Center for Hybrid Wheat, Beijing Academy of Agriculture and Forestry \\ Sciences, Beijing 100097, China; gshiq@126.com \\ * Correspondence: mayouzhi@caas.cn (Y.M.); chenming02@caas.cn (M.C.) \\ + Equally contributed.
}

check for updates

Citation: Qi, X.; Tang, W.; Li, W.; He, Z.; Xu, W.; Fan, Z.; Zhou, Y.; Wang, C.; $\mathrm{Xu}, \mathrm{Z}$.; Chen, J.; et al. Arabidopsis G-Protein $\beta$ Subunit AGB1 Negatively Regulates DNA Binding of MYB62, a Suppressor in the Gibberellin Pathway. Int. J. Mol. Sci. 2021, 22, 8270. https://doi.org/ $10.3390 /$ ijms 22158270

Academic Editor: Karen Skriver

Received: 25 May 2021

Accepted: 27 July 2021

Published: 31 July 2021

Publisher's Note: MDPI stays neutral with regard to jurisdictional claims in published maps and institutional affiliations.

Copyright: (c) 2021 by the authors. Licensee MDPI, Basel, Switzerland. This article is an open access article distributed under the terms and conditions of the Creative Commons Attribution (CC BY) license (https:/ / creativecommons.org/licenses/by/ $4.0 /)$.
Abstract: Plant $G$ proteins are versatile components of transmembrane signaling transduction pathways. The deficient mutant of heterotrimeric $G$ protein leads to defects in plant growth and development, suggesting that it regulates the GA pathway in Arabidopsis. However, the molecular mechanism of $G$ protein regulation of the GA pathway is not understood in plants. In this study, two G protein $\beta$ subunit (AGB1) mutants, agb1-2 and N692967, were dwarfed after exogenous application of $\mathrm{GA}_{3}$. AGB1 interacts with the DNA-binding domain MYB62, a GA pathway suppressor. Transgenic plants were obtained through overexpression of MYB62 in two backgrounds including the wild-type (MYB62/WT Col-0) and agb1 mutants (MYB62/agb1) in Arabidopsis. Genetic analysis showed that under $\mathrm{GA}_{3}$ treatment, the height of the transgenic plants $M Y B 62 / W T$ and $M Y B 62 / a g b 1$ was lower than that of WT. The height of MYB62/agb1 plants was closer to MYB62/WT plants and higher than that of mutants agb1-2 and N692967, suggesting that MYB62 is downstream of AGB1 in the GA pathway. qRT-PCR and competitive DNA binding assays indicated that MYB62 can bind MYB elements in the promoter of $G A 20 x 7$, a GA degradation gene, to activate GA2ox7 transcription. AGB1 affected binding of MYB62 on the promoter of GA2ox7, thereby negatively regulating th eactivity of MYB62.

Keywords: Arabidopsis; GA signaling; AGB1; MYB62; protein interaction

\section{Introduction}

The heterotrimeric $G$ protein pathway is a conservative transmembrane signal transduction pathway found in both animals and plants [1,2]. In Arabidopsis, the heterotrimeric $\mathrm{G}$ protein is composed of an $\alpha$ subunit (GPA1), a $\beta$ subunit (AGB1), and three $\gamma$ subunits (AGG1, AGG2, and AGG3) [3]. During G protein signaling transduction, G- $\alpha$ binds to GDP and forms a coupling polymer with the $G-\beta-\gamma$ dimer, leaving the $G$ protein pathway in a resting state [4] (Anantharaman et al., 2011). When the G protein pathway is activated, the GTP-G- $\alpha$ monomer is dissociated from the G- $\beta-\gamma$ dimer, while the GTP-G- $\alpha$ monomer and the G- $\beta-\gamma$ dimer interact with a variety of downstream effectors to transmit signals for different cell and physiological functions [4] (Anantharaman et al., 2011). The heterotrimeric $\mathrm{G}$ proteins are involved in growth and developmental processes such as seed 
germination and seedling development [5] (Ullah et al., 2003), cell division and morphology [6] (Ullah et al., 2002), ion channel regulation [7] (Assmann and Yu, 2015), stomatal development [8] (Wang et al., 2011), and the response to environmental conditions such as phytohormones, sugar, ROS (reactive oxygen species), and light [9] (Li et al., 2012). Plants with mutated $G$ protein complex components have altered morphology in their fruits, grain weight, roots, and leaves, and these mutants are sensitive to a variety of hormones, including IAA (auxin), GA (gibberellins), and BR (brassinosteroids) [2,5,10-12] (Ullah et al., 2003; Chen et al., 2004; Pandey et al., 2006; Chen, 2007; Urano et al., 2014).

The G- $\alpha$ subunit positively regulates the GA pathway by inhibiting the activity of SLR (slender rice), a negative regulator of GA signaling in rice [13] (Ueguchi-Tanka et al., 2000), and the Arabidopsis G- $\alpha$ mutant gpa1 is less sensitive to GA [14] (Trusov et al., 2007). The G- $\beta$ subunit is involved in a variety of signaling pathways in plants. In Arabidopsis, the interactions between AGB1 and ERECTA during silique development were the first evidence of the interaction between $G$ proteins and receptor kinases [15] (Lease et al., 2001). We previously found that AGB1 is involved in regulating ABA and drought response by interacting with the protein kinase MPK6 and the transcription factor VIP1 [16] (Xu et al., 2015). We also found that AGB1 interacts with the transcription factor BBX21 to regulate photomorphogenesis in Arabidopsis [17] (Xu et al., 2017). In rice, suppression of the G- $\beta$ subunit gene, RGB1, causes dwarfism and browning of the internodes and lamina joint regions [18] (Utsunomiya et al., 2011). In Arabidopsis, the G- $\beta$ subunit gene mutant agb1 has shorter mature plants than the wild-type (WT) [19] (Urano et al., 2016). These results suggest that the G- $\beta$ subunit also regulates plant development through the GA pathway, but the specific mechanism is not clear.

GA functions directly in regulating plant growth and development as well as crop yield [20] (Singh et al., 2002). GA-related genes can be divided into two categories: either the pathways involved in GA synthesis or degradation, or the GA signaling transduction pathway. In plants, a variety of enzymes are involved in GA synthesis, while GGPP (geranylgeranyl pyrophosphate), a precursor of GA synthesis, is catalyzed by GGPS (geranylgeranyl pyrophosphate synthase) [21] (Lange et al., 2003). GGPP is further catalyzed by CPS (copalyl pyrophosphate synthase) and KS (ent-kaurene synthase), which forms kaurene [22] (Morrone et al., 2009). Additionally, KO (ent-kaurene oxidase) and KAO (ent-kaurenoic acid oxidase) have important roles in the GA synthesis intermediate $\mathrm{GA}_{12^{-}}$ aldehyde [23,24] (Davidson et al., 2003; Sakamoto et al., 2004). GA 12 -aldehyde is a branch point of GA, which hydroxylates to form $\mathrm{GA}_{12}$ and $\mathrm{GA}_{53}$ under the oxidation of GA20ox1 (gibberellin 20-oxidase gene). $\mathrm{GA}_{12}$ and $\mathrm{GA}_{53}$ produce $\mathrm{GA}_{9}$ and $\mathrm{GA}_{20}$, while the final form of bioactive GA is catalyzed by GA30ox1 [25] (Hedden et al., 2012).

In rice, the plant is dwarfed due to the mutation of GA20ox, a key gene for GA synthesis [26] (Ashikari et al., 2002). The deletion of $K A O$ can lead to severe dwarfing in many plants, such as rice ( $d 35$ mutant) [24] (Sakamoto et al., 2004), maize (dwarf3) [27] (Helliwell et al., 2001), pea (na) [23] (Davidson et al., 2003), and sunflower (dwarf2) [28] (Fambrini et al., 2011). The signal transduction of GA in plants is mediated by the receptor GID1, while DELLA is an inhibitor of the GA pathway via transcriptional regulation [29] (Ueguchi-Tanaka et al., 2005). GA promotes the formation of the GA-GID1DELLA complex via conformational changes caused by GID1 binding, where the DELLA protein is then ubiquitinated and degraded by the $26 \mathrm{~S}$ proteasome to open the GA pathway [30,31] (Murase et al., 2008; Shimada et al., 2008). In Arabidopsis, the transcription factor MYB62 is involved in the GA pathway, and overexpression of MYB62 results in a GAdeficient phenotype, which suggests that MYB62 is a suppressor in the GA pathway [32] (Devaiah et al., 2009).

In this study, we found that plant heights in two G- $\beta$ subunit mutants, agb1-2 and N692967, were significantly lower than in the WT following GA $_{3}$ treatment, suggesting that the function of AGB1 in the GA pathway is similar to that of GPA1 in Arabidopsis. We found that AGB1 regulates the GA pathway by negatively regulating the DNA binding of MYB62, a GA pathway suppressor on the promoter of the GA degradation gene GA2ox7. 
The G protein complex regulates the GA pathway through the AGB1-MYB62-GA2ox7 pair in Arabidopsis.

\section{Materials and Methods}

\subsection{Plant Materials and Growth Conditions}

The agb1 mutant agb1-2 (CS6536) has been described by Ullah et al. (2003). In the other agb1 mutant N692967 (SALK_204268C), the T-DNA insert occurs in chr4 16,477,780 of the Col-0 genome (Supplemental Figure S1). The expression of AGB1 in mutants and MYB62 in transgenic plants was identified by qRT-PCR using gene-specific primers (Table S1, Supplemental Figure S1). Because we could not obtain MYB62 mutants, we overexpressed the $M Y B 62$ gene in different genetic backgrounds to obtain transgenic plants, including $M Y B 62$ transgenic plants in a WT background-MYB62:GFP/WT-8 and MYB62:GFP/WT-10 - and MYB62 transgenic plants in an agb1 background-MYB62:GFP/agb1-2-1, MYB62:GFP/agb12-4. The response of Arabidopsis plants to $\mathrm{GA}_{3}$ treatment was observed at the seedling stage. Before planting Arabidopsis (Col-0) seeds on plates, the seeds were soaked with $10 \%$ sodium hypochlorite for $10 \mathrm{~min}$ and then washed 3 times with sterile distilled water. The sterilized Arabidopsis seeds were treated at $4{ }^{\circ} \mathrm{C}$ in the dark for 3 days and then germinated on $1 / 2$ MS medium ( $0.8 \%$ agar) for 7 days. When Arabidopsis grew to the 4-leaf stage, seedlings were transplanted into $1 / 2 \mathrm{MS}$ medium containing $1 \mu \mathrm{M} \mathrm{GA}_{3}, 10 \mu \mathrm{M} \mathrm{GA}_{3}$, or $100 \mu \mathrm{M} \mathrm{GA}_{3}$ and grown at $24{ }^{\circ} \mathrm{C}$ in the light for $16 \mathrm{~h}$ then at $20^{\circ} \mathrm{C}$ in darkness for $8 \mathrm{~h}$ (Supplemental Figures S2-S5). The growth state was observed and recorded every day. Differences were observed when the seedings were grown under $\mathrm{GA}_{3}$ treatment for 10 days.

\subsection{Measurement of Gibberellin Content}

Total GA content, including $\mathrm{GA}_{1}, \mathrm{GA}_{2}, \mathrm{GA}_{3}, \mathrm{GA}_{4}, \mathrm{GA}_{7}$, and $\mathrm{GA}_{20}$, was measured using an ELISA kit (Plant GA ELISA Kit, X-Y Biotechnology company) according to the manufacturer's instructions. Plant samples $(0.1 \mathrm{~g})$ were ground, then dissolved in $900 \mu \mathrm{L}$ of PBS buffer, fully mixed and centrifuged at $12,000 \mathrm{rpm}$ for $10 \mathrm{~min}$ at $4{ }^{\circ} \mathrm{C}$. All standards and samples were added in duplicate to Micro-ELISA strip plate wells (Eppendorf, Germany). The volume for a standard curve sample and a measured sample was $50 \mu \mathrm{L}$, and nothing was added to the blank well. A total of $100 \mu \mathrm{L}$ of HRP-conjugate reagent was added to each well, then the wells were covered with tinfoil and incubated for $60 \mathrm{~min}$ at $37^{\circ} \mathrm{C}$. After washing 5 times, $50 \mu \mathrm{L}$ of chromogen Solution A and $50 \mu \mathrm{L}$ of chromogen Solution B were added to each well. The plate was incubated for $15 \mathrm{~min}$ at $37^{\circ} \mathrm{C}$ and $50 \mu \mathrm{L}$ of a stop solution was added to each well. The optical density at $450 \mathrm{~nm}$ was read using a microtiter plate within $15 \mathrm{~min}$. The standard curve was generated by plotting the average $\mathrm{OD}_{450}$ obtained for each of the 6 standard concentrations on the vertical $(y)$ axis versus the corresponding concentration on the horizontal $(x)$ axis. The concentration of the sample was calculated according to the equation of the standard curve.

\subsection{Construction of Plasmids and Transgenic Lines}

The full length of MYB62 (AT1G68320) was amplified using the primers 1302-MYB62-F and 1302-MYB62-R and inserted into the pCambia-1302 vector (Clontech, San Francisco, CA, USA) with GFP at the C-terminal end through the BamHI restriction enzyme cutting site. The constructs were transformed into wild-type (Col-0) and mutant Arabidopsis agb1-2 at the flowering stage by Agrobacterium tumefaciens (GV3101)-mediated transformation [33] (Clough et al., 1998). The seeds of the T0 generation of transgenic Arabidopsis were sown on selective medium (MS medium with $40 \mathrm{mg} / \mathrm{L}$ hygromycin), and the seedings were transplanted into the soil in pots. The seeds of theT1 and T2 transgenic lines were further screened by hygromycin. More than $95 \%$ of the seeds of the T2 generation with hygromycin resistance were homozygous lines. The MYB62:GFP/agb1-2 transgenic plants were identified using kanamycin and hygromycin for screening and identification. The phenotypes of homozygous lines were analyzed under $\mathrm{GA}_{3}$ treatment in the $\mathrm{T} 3$ generation. 


\subsection{Extraction of RNA and Analysis of Gene Expression}

Seedlings of MYB62 transgenic lines and the mutants agb1-2 and N692967, and the roots and leaves of WT Arabidopsis were used for gene expression analysis. The total plant RNA was extracted using the Trizol method (Zhuangmeng Total RNA Extraction Kit), and the RNA was reverse-transcribed into cDNA using the TransScript One-Step gDNA removal and cDNA Synthesis SuperMix kit (TransGen Biotech, Beijing, China). We performed qRT-PCR using cDNA as the template and the primers in Table S1 according to the Real Master Mix (SYBR Green) kit (TransGen Biotech, Beijing, China). Gene expression was calculated using the $2^{-\Delta \Delta C T}$ method [34] (Livak \& Schmittgen, 2001). Relative quantitative results were calculated through normalization based on the control gene ACT2 (AT3G18780).

\subsection{Subcellular Localization Analysis}

The subcellular localization of MYB62 protein was completed in WT Col-0 Arabidopsis protoplasts. Protoplasts were prepared from the wild-type seedling leaves of Arabidopsis before bolting, according to the methods used in a previous study [35] (Yoo, Cho, \& Sheen, 2007). The full-length coding sequences of $A G B 1$ and MYB62 were amplified using gene-specific primers (Table S1), and $A G B 1$ and $M Y B 62$ were inserted into the vector 16318h-GFP to express the fused proteins GFP-AGB1 and GFP-MYB62, respectively. The vectors 16318-AGB1-GFP and 16318-MYB62-GFP were then separately transformed into protoplasts. A confocal laser scanning microscope (LSM700, Zeiss, Yena, Germany) was used to observe the experimental results.

\subsection{Yeast Two-Hybrid Assay}

To analyze the interaction between AGB1 and MYB62, AGB1 and MYB62 were, respectively, inserted into the pGADT7 vector and the pGBKT7 vector in the EcoR1 and BamH1 restriction enzyme cutting sites. We prepared the yeast cells and completed vector transformation according to the manufacturer's instructions (TaKaRa, Tokyo, Japan). The transformants were selected on a synthetic dextrose (SD) medium lacking leucine and tryptophan (SD/-Leu/-Trp). The yeast transformants from the SD (-Leu/-Trp) were then streaked onto a solid SD (-Leu/-Trp/-His/-Ade) medium, with or without $40 \mu \mathrm{g} / \mathrm{mL}$ $\mathrm{X}$ - $\alpha$-gal, to observe and photograph their growth.

\subsection{Bimolecular Fluorescence Complementation (BiFC) Assay}

AGB1 and MYB62 were, respectively, fused to the $\mathrm{N}$ - and C-termini of the luciferase reporter gene LUC, while the constructed vector was transformed into a strain of A. tumefaciens, GV3101. The A. tumefaciens samples transformed with nLUC-AGB1 and MYB62-cLUC were selected and the OD value of $A$. tumefaciens was adjusted to 0.8 with the infection solution ( $10 \mathrm{mM}$ MES, $150 \mu \mathrm{M}$ AS, $10 \mathrm{mM} \mathrm{MgCl}{ }_{2} 6 \mathrm{H}_{2} \mathrm{O}$ ). The $A$. tumefaciens samples transformed using the control, nLUC-AGB1, and MYB62-cLUC were mixed as pairs of nLUC and $C L U C, n L U C-A G B 1$ and $c L U C, n L U C$ and MYB62-cLUC, and nLUC-AGB1 and MYB62-cLUC, then injected into Nicotiana benthamiana leaves. Before analyzing LUC activity, N. benthamiana was cultured in darkness for $72 \mathrm{~h}$. We performed 3 biological replicates for each combination of nLUC- and cLUC-infected tobacco leaves as a control.

\subsection{Pull-Down Assays}

MYB62 was inserted into the pMAL-c2x (MBP-Tag) vector to express MBP-labeled fusion proteins (Takara, Japan), and AGB1 was inserted into the pGEX4T-1 (GST-Tag) vector to express GST-labeled fusion proteins (Takara, Tokyo, Japan). The vectors pMAL-c2xMYB62 and pGEX4T-1-AGB1 were then introduced into Escherichia coli BL21 (DE3). The expression of GST and MBP fusion proteins was induced by isopropylthio- $\beta$-galactoside (IPTG) and expressed at $16^{\circ} \mathrm{C}$ for a minimum of $16 \mathrm{~h}$. The fusion proteins of GST-AGB1 and MBP-MYB62 were purified by glutathione-agarose 4B (GE Healthcare, Stockholm, Sweden) beads and MBP-agarose gel, according to the instructions of the manufacturer. 
In total, $50 \mu \mathrm{L}$ of each recombinant fusion protein was mixed with $1 \mathrm{~mL}$ of the binding buffer ( $40 \mathrm{mM}$ HEPES, $10 \mathrm{mM} \mathrm{KCl}, 0.4 \mathrm{M}$ sucrose, $3 \mathrm{mM} \mathrm{MgCl}_{2} 6 \mathrm{H}_{2} \mathrm{O}, 1 \mathrm{mM}$ EDTA, $1 \mathrm{mM}$ DTT) in the pull-down assay. After eluting the MYB62 protein from the MBP-agarose gel with a $10 \mu \mathrm{M}$ maltose solution, the MYB62-MBP protein and the GST-AGB1 protein were incubated in a pull-down buffer for approximately $8 \mathrm{~h}$ at $4{ }^{\circ} \mathrm{C}$. They were then centrifuged at $2000 \times g$ for $1 \mathrm{~min}$ and washed 5 times at $4{ }^{\circ} \mathrm{C}$ with a $1 \times$ PBS buffer (pH 7.4). The particles containing binding proteins were then boiled in a $1 \times$ PBS buffer, and the released proteins were separated with $10 \%$ SDS-PAGE. The antibodies MBP-Tag and GST Tag were analyzed using a Western blot analysis (Abcam, Cambridge, UK).

\subsection{Transcriptional Activation Experiment in Yeast}

The transcription activation experiment was performed according to the methods used in previous research [36] (Yamaji et al., 2009). In order to further explore whether AGB1 affects the transcriptional activation of $M Y B 62$, we carried out transcriptional activation experiments in yeast cells (Figure 5B). MYB62 was inserted into pBridge vector to construct pBridge-MYB62, and MYB62 was fused with the binding domain of the GAL4 transcription factor (GAL4-BD), which can bind target sequences upstream of the reporter genes (histidine-deficient reporter gene) in yeast chromosomes. When MYB62 was inserted into pBridge, the reporter gene could be activated depending on the transcriptional activation activity of MYB62 (Figure 5B). The yeast transformed with the pBridge-MYB62 vector could grow on the screening medium. When $A G B 1$ was inserted into another expression cassette of the pBridge-MYB62 vector to make pBridge-MYB62-AGB1, the effect of AGB1 on the transcriptional activation of MYB62 could be detected (Figure 5B). These constructed bodies were then introduced into the yeast reporter strain AH109 (Yeast Protocols Handbook; TaKaRa, Japan). The transformed yeast cells were selected on the selective medium (SD/-Trp, SD/-Trp-Ade, SD/-Trp-His-Ade) and the growth status was recorded.

\subsection{LUC Assay of MYB62 in Tobacco (N. benthamiana)}

MYB62 was inserted into the pCambia-1302 vector as an effector vector, and the 2000 bp promoter sequences of the downstream genes, including GA2ox7, were fused into the pGreenII 0800-LUC vector as a reporter vector. The reporter gene and the effector gene were introduced into GV3101, a strain of A. tumefaciens, and then injected into tobacco leaves. The activity of $L U C$ was observed after $72 \mathrm{~h}$ of growth. Each sample was injected into 8 tobacco leaves, with 3 biological replicates performed for each.

\subsection{Electrophoretic Mobility Shift Assay (EMSA)}

MYB62-MBP and AGB1-GST proteins were induced by IPTG in E. coli BL21 (DE3). The fusion proteins of GST-AGB1 and MBP-MYB62 were purified by glutathione-agarose 4B (GE Healthcare, North Richland Hills, TX, USA) beads and MBP-agarose gel, according to the instructions of the manufacturer. The synthetic oligonucleotide probe was synthesized by the ShengGong Biotech Company. The LightShift chemiluminescence EMSA kit (Thermo Science, Waltham, MA, USA) was used for EMSA. The biotin-labeled probe was incubated for $30 \mathrm{~min}$ at room temperature in a binding buffer $(2.5 \%$ glycerol, $50 \mathrm{mM} \mathrm{KCl}, 5 \mathrm{mM}$ $\mathrm{MgCl}_{2}$, and $10 \mathrm{mM}$ EDTA) with or without MYB62-MBP or AGB1-GST fused protein. For unlabeled probe competition, an unlabeled probe was added to the reaction, and single GST and MBP tags were used as negative controls. The probe sequence is shown in Table S1.

\subsection{Low Phosphate-Tolerant Phenotypic Assay of Plants}

Sterilized Arabidopsis seeds were placed in a 1/2 MS medium and cultured in an incubator $\left(24{ }^{\circ} \mathrm{C} / 16 \mathrm{~h}, 20^{\circ} \mathrm{C} / 8 \mathrm{~h}\right)$. The seedlings were transplanted into a $1 / 2 \mathrm{MS}$ medium (Caissonabs, Rexburg, ID, USA) and a phosphate-free medium (Caissonabs, USA); the media's formulations are shown in Tables $\mathrm{S} 2$ and S3, respectively. The seedlings were then cultured in an incubator $\left(24{ }^{\circ} \mathrm{C} / 16 \mathrm{~h}, 20^{\circ} \mathrm{C} / 8 \mathrm{~h}\right)$ for 7-10 days. 


\section{Results}

3.1. AGB1 Mutants agb1-2 and N692967 Were Dwarfed Compared with the WT after Exogenous Application of $\mathrm{GA}_{3}$

After treatment with $10 \mu \mathrm{M} \mathrm{GA}$, we found that the plant height of agb1-2 was lower than that of the WT (Supplementary Figure S2). In addition, we identified another homozygous AGB1 mutant, N692967 (SALK_204268C) (Supplementary Figure S1A), and carried out phenotypic experiments under te conditions of $1 \mu \mathrm{M} \mathrm{GA}_{3}, 10 \mu \mathrm{M} \mathrm{GA}_{3}$, or $100 \mu \mathrm{M} \mathrm{GA} 3$ treatment (Supplementary Figures S3-S5). Without $\mathrm{GA}_{3}$ treatment, plant height and the rosette leaf of the mutants agb1-2 and N692967 were slightly smaller than in the WT (Figure 1A,B). When treated with different concentrations of $\mathrm{GA}_{3}$ for 10 days, the plant height of the mutants agb1-2 and N692967 was significantly lower than that of the WT (Figure 1D,E and Figures S3-S5). Moreover, we found that following an increase in $\mathrm{GA}_{3}$ concentration, the plant height of $A G B 1$ mutant partially recovered compared with the WT, indicating that $A G B 1$ may be involved in the GA synthesis or degradation pathway. Therefore, in order to analyze the downstream pathway of $A G B 1$, we analyzed the endogenous GA content of the AGB1 mutant. The results showed that without $\mathrm{GA}_{3}$ treatment, there were no significant differences in GA content between the mutants agb1-2 and N692967, and the WT (Figure 1C), but under GA 3 treatment, the GA contents of agb1-2 and N692967 were significantly lower than that of the WT (Figure 1F), consistent with the results of their height (Figure 1B,E).
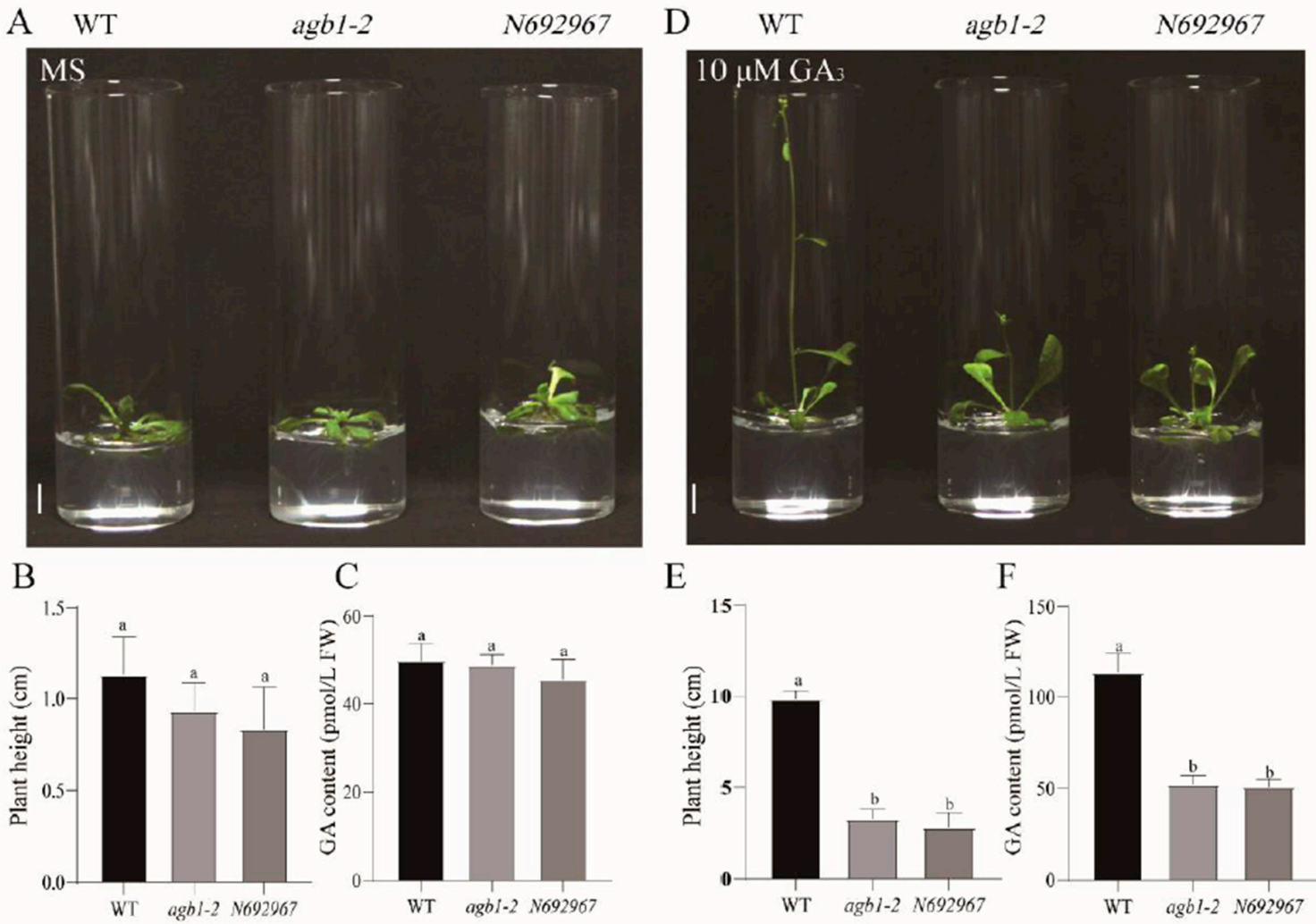

Figure 1. Phenotypes of the wild-type (Col-0), agb1-2, and N692967 under GA 3 treatment. (A) Plant height phenotypes of the wild-type (Col-0), agb1-2, and N692967 under normal conditions. Scale bars, $1 \mathrm{~cm}$. (B,C) Plant height and GA content of the wild-type (Col-0), agb1-2, and N692967 under normal conditions. Data are the average of three independent experiments, and the error bars represent SE $(n=10)$. Significant differences were analyzed using Duncan's multiple range test $(p<0.05)$. (D) Plant height phenotypes of the wild-type (Col-0), agb1-2, and N692967 treated with $10 \mu \mathrm{M} \mathrm{GA}$. Scale bars, $1 \mathrm{~cm}$. (E,F) Plant height and GA content of the wild-type (Col-0), agb1-2, and N692967 under normal conditions. Data are the average of three independent experiments, and the error bar represents the SE $(n=10)$. Significant differences were analyzed using Duncan's multiple range test $(p<0.05)$. 


\subsection{AGB1 Interacts with the DNA-Binding Region of MYB62, a GA Pathway Suppressor}

Overexpression of MYB62 reduced the sensitivity of plants to GA treatment and reduced tolerance to low-phosphorus stress [32] (Devaiah, Madhuvanthi, Karthikeyan, \& Raghothama, 2009). This phenotype, under $\mathrm{GA}_{3}$ treatment and low-phosphorus stress, was similar to that of the agb1-2 mutant in our study (Supplementary Figures S6-S8 and S11). Therefore, we tried to identify the interaction between AGB1 and MYB62 using a yeast two-hybrid assay. The yeast cells grew on selective media (SD/-Trp/-Leu/-His/-Ade) and selective media plus $x-\alpha$-gels only when BD-AGB1 and AD-MYB62 fused proteins were co-expressed in yeast cells (Figure 2A). The pull-down experiment demonstrated that AGB1 and MYB62 interacted in vitro (Figure 2B). The firefly luciferase (LUC) complementary imaging (Lci) analysis demonstrated that when AGB1-nLUC and MYB62-cLUC were expressed in the leaves of $N$. benthamiana, strong LUC activity was observed, whereas there was no LUC activity in the negative control (including nLUC + cLUC, AGB1-nLUC + cLUC, and nLUC + MYB62-cLUC) (Figure 2C). The quantitative analysis results of LUC activity (Figure 2D) were consistent with those shown in Figure 2C. These results demonstrated that AGB1 can interact with MYB62 in plant cells.

A
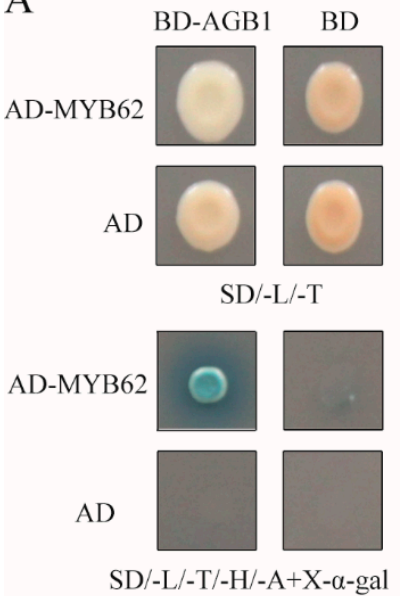

B

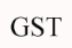

GST-AGB1 MBP-MYB62

Pull down Anti-MBP

Input Anti-GST

Input Anti-MBP

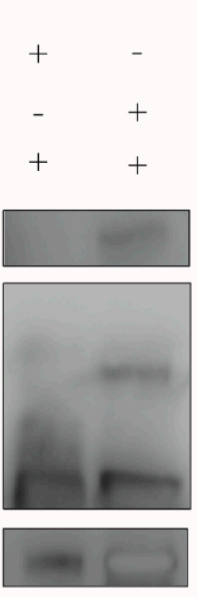

$\mathrm{C}$

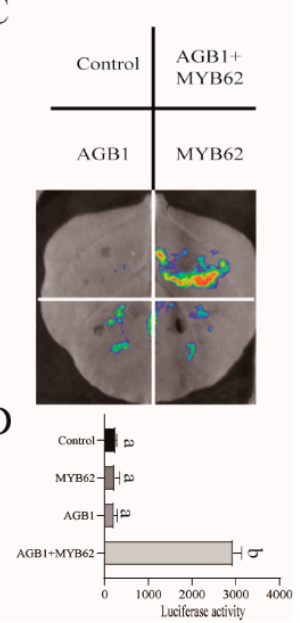

Figure 2. The interaction between AGB1 and MYB62. (A) Yeast two-hybrid interactions between full-length sequences of $A G B 1$ and $M Y B 62$. The transformed yeast cells were activated and cultured on SD/-Leu/-Trp and SD/-Leu/-Trp/-His/-Ade media. The yeast cells of the empty vectors AD and BD were used as negative controls. (B) Protein interaction of AGB1 and MYB62. In vitro GST pull-down assays showed that AGB1 interacted with MYB62 in vitro. (C) Interaction of AGB1 with MYB62. A luciferase (LUC) assay was performed to demonstrate that AGB1 and MYB62 can interact with $N$. benthamiana leaf cells. AGB1 and MYB62 were fused with nLUC and cLUC, respectively. nLUC-only and cLUC-only refer to the empty vectors used as negative controls. (D) Quantification of luminous intensity in C. Error bars represent the means \pm SE $(n=3)$. Significant differences were analyzed using Duncan's multiple range test $(p<0.05)$.

To identify the interaction regions of MYB62, we inserted three truncated (M1-M3) segments as well as full-length MYB62 into the AD vectors and inserted AGB1 into the BD vector. These vectors were transformed into yeast cells to identify the interactions between different regions of MYB62 and AGB1. Our results demonstrated that full-length MYB62 interacted with AGB1, and the M1 and M2 truncated structures interacted slightly with AGB1, while M3 did not interact with AGB1 (Figure 3A), which suggests that the two DNA-binding regions of MYB62 were necessary for the interaction with AGB1. 

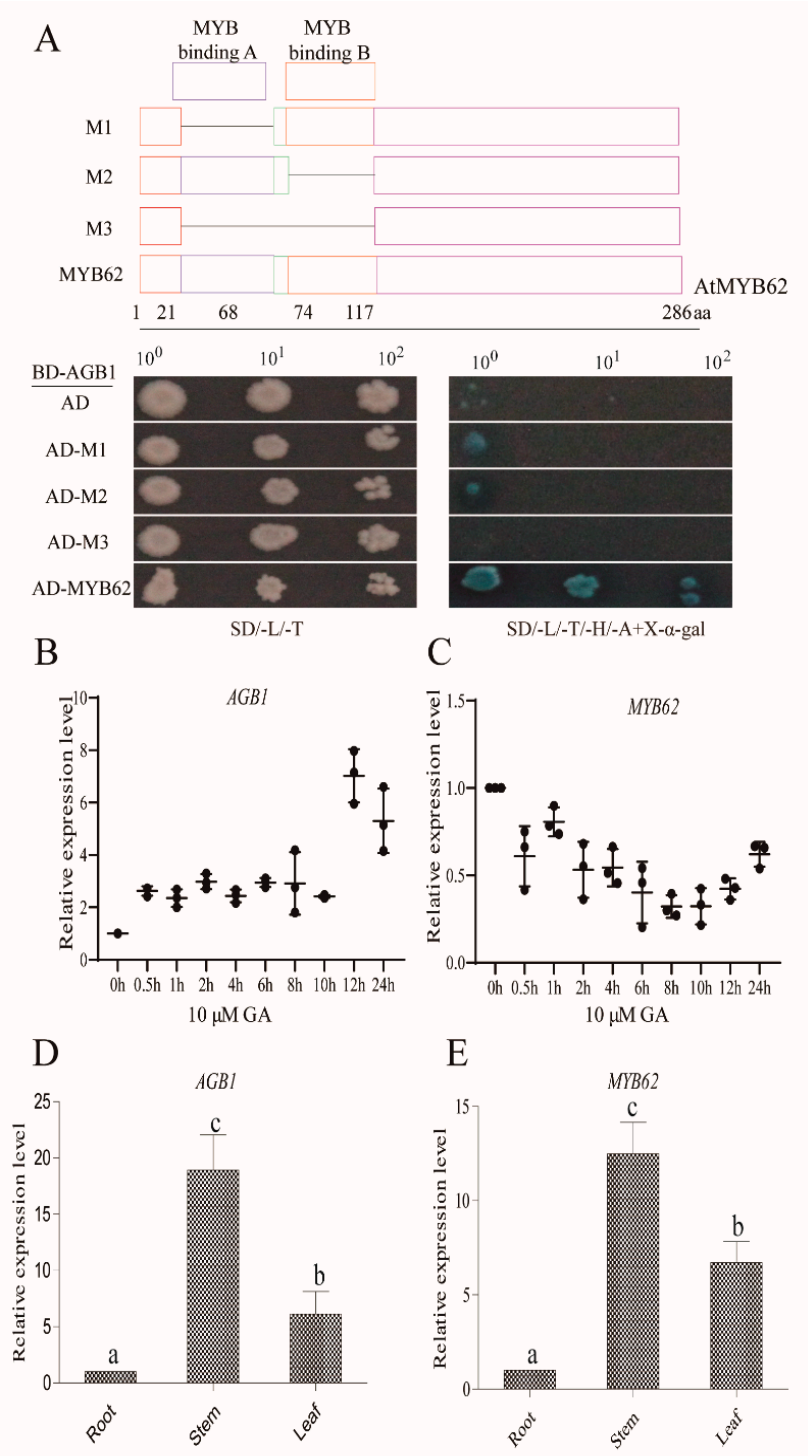

Figure 3. AGB1 and MYB62 expression analysis. (A) Yeast two-hybrid interactions between the fulllength sequence of AGB1 and the piecewise sequence of MYB62. The transformed yeast cells were activated and cultured on SD/-Leu/-Trp and SD/-Leu/-Trp/-His/-Ade media. The yeast cells of the empty vectors $\mathrm{AD}$ and $\mathrm{BD}$ were used as negative controls. (B) Analysis of AGB1 expression in wild-type Col-0 leaves grown for 4 weeks after treatment with $10 \mu \mathrm{M} \mathrm{GA} 3$ at different time periods. The data are the average of three independent experiments, and the error bar represents the SE $(n=3)$. Significant differences were analyzed using Duncan's multiple range test $(p<0.05)$. Relative quantitative results were calculated by normalization using the control gene ACT2 (AT3G18780). (C) Analysis of MYB62 expression in wild-type Col-0 leaves, grown for 4 weeks after treatment with $10 \mu \mathrm{M}$ GA3 at different time periods. The data are the average of three independent experiments, and the error bar represents the SE $(n=3)$. Significant differences were analyzed using Duncan's multiple range test $(p<0.05)$. (D) Analysis of AGB1 expression in the roots, stem, and leaves of wild-type Col-0 grown for 4 weeks under normal growth conditions. The data are the average of three independent experiments, and the error bar represents the SE $(n=3)$. a, b c indicate significant differences. Significant differences were analyzed using Duncan's multiple range test $(p<0.05)$. (E) Analysis of MYB62 expression in the roots, stem, and leaves of wild-type Col-0 grown for 4 weeks under normal growth conditions. The data are the average of three independent experiments, and the error bar represents the SE $(n=3)$. Significant differences were analyzed using Duncan's multiple range test $(p<0.05)$. 
In addition, in seedlings of WT Arabidopsis, the expression of AGB1 was induced under $\mathrm{GA}_{3}$ treatment (Figure $3 \mathrm{~B}$ ), whereas the expression of $M Y B 62$ was inhibited under $\mathrm{GA}_{3}$ treatment (Figure 3C). The tissue-specific expression of $A G B 1$ and MYB62 at the seedling stage was analyzed in Arabidopsis (Col-0) without $\mathrm{GA}_{3}$ treatment. The results showed that MYB62 was mainly expressed in Arabidopsis stems, which was similar to AGB1 (Figure 3D,E). These results indicate that both $A G B 1$ and MYB62 play an important role in stem development.

\subsection{Genetic Analysis Indicated that MYB62 Was Downstream of AGB1 in the GA Pathway}

In previous studies, researchers hoped to obtain homozygous mutants of $M Y B 62$ by T-DNA insertion and antisense and RNAi-mediated MYB62 silencing, but were unsuccessful and thus created the MYB62 overexpression plant [32] (Devaiah, Madhuvanthi, Karthikeyan, \& Raghothama, 2009). In order to study the genetic relationship between AGB1 and MYB62 further, we separately overexpressed MYB62 in WT Arabidopsis (MYB62:GFP/WT-8 and MYB62:GFP/WT-10) and agb1-2 (MYB62:GFP/agb1-2-1 and MYB62:GFP/agb1-2-4). Phenotypic analyses were carried out under the conditions of $1 \mu \mathrm{M} \mathrm{GA}_{3}, 10 \mu \mathrm{M} \mathrm{GA}_{3}$, and $100 \mu \mathrm{M} \mathrm{GA}_{3}$, and the plant height was recorded (Figure 4 and Figures $\mathrm{S6}-\mathrm{S} 8$ ). Phenotypic analysis showed that without $\mathrm{GA}_{3}$ treatment, the mutants agb1-2 and N692967, and the transgenic plants MYB62:GFP/WT-8, MYB62:GFP/WT10, MYB62:GFP/agb1-2-1, and MYB62:GFP/agb1-2-4 were slightly smaller than the WT (Figure 4A). The GA content of MYB62:GFP/WT-8, MYB62:GFP/WT-10, MYB62:GFP/agb1-21, and MYB62:GFP/agb1-2-4 was significantly lower than that of the WT and AGB1 mutants (Figure 4C).

Under $10 \mu \mathrm{M} \mathrm{GA}_{3}$ treatment, the height and the flowering time of the mutants agb1-2 and N692967 and the transgenic Arabidopsis, including MYB62:GFP/WT-8, MYB62:GFP/WT-10, MYB62:GFP/agb1-2-1, and MYB62:GFP/agb1-2-4, were significantly lower than in the WT (Figure 4D,E). The GA contents of the mutants agb-1-2 and $N 692967$ and transgenic Arabidopsis, including MYB62:GFP/WT-8, MYB62:GFP/WT-10, MYB62:GFP/agb1-2-1, and MYB62:GFP/agb1-2-4 were lower than that of the WT (Figure 4F), consistent with their height results (Figure 4D,E). In addition, the plant height of MYB62:GFP/agb1-2 transgenic plants were more similar to that of MYB62:GFP/WT, and was higher than in the mutants agb-1-2 and N692967, also consistent with their GA content results (Figure 4D-F). The height and GA content analysis was carried out under $1 \mu \mathrm{M} \mathrm{GA}_{3}$ and $100 \mu \mathrm{M} \mathrm{GA}_{3}$, and the results were consistent with those under $10 \mu \mathrm{M} \mathrm{GA}_{3}$ (Supplementary Figures S9 and S10). The genetic analysis showed that Arabidopsis MYB62 and AGB1 belong to the same GA pathway, and MYB62 is downstream of $A G B 1$ in this GA pathway. 


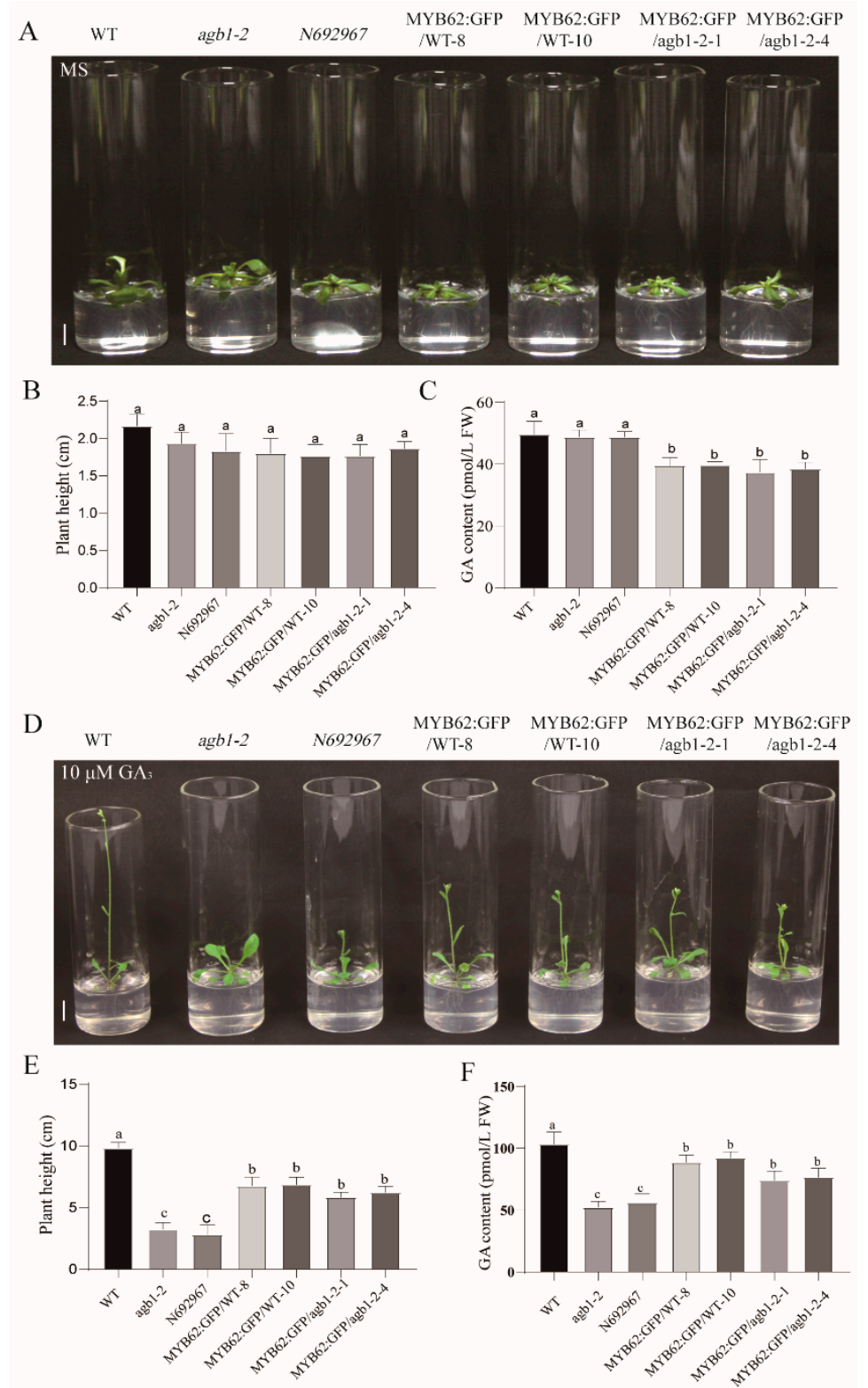

Figure 4. Phenotypic identification of the wild-type, agb-1-2, N692967, MYB62:GFP/WT-8, MYB62:GFP/WT-10, MYB62:GFP/agb1-2-1, and MYB62:GFP/agb1-2-4 under normal conditions and $10 \mu \mathrm{M} \mathrm{GA} 3$ treatment. (A) Plant height phenotype of the wild-type (Col-0), agb-1-2, N692967, MYB62:GFP/WT-8, MYB62:GFP/WT-10, MYB62:GFP/agb1-2-1, and MYB62:GFP/agb1-2-4 under normal conditions. Scale bars, $1 \mathrm{~cm}$. (B,C) Plant height and GA content of the wild-type (Col-0), agb-1-2, N692967, MYB62:GFP/WT-8, MYB62:GFP/WT-10, MYB62:GFP/agb1-2-1, and MYB62:GFP/agb1-2-4 under normal conditions. The data are the average of three independent experiments, and the error bar represents the SE $(n=10)$. a, b indicate significant differences. Significant differences were analyzed using Duncan's multiple range test $(p<0.05)$. (D) Plant height phenotype of the wildtype (Col-0), agb-1-2, N692967, MYB62:GFP/WT-8, MYB62:GFP/WT-10, MYB62:GFP/agb1-2-1, and MYB62:GFP/agb1-2-4 under $10 \mu \mathrm{MGA}_{3}$ treatment. Scale bars, $1 \mathrm{~cm}$. (E,F) Plant height and GA content of the wild-type (Col-0), agb-1-2, N692967, MYB62:GFP/WT-8, MYB62:GFP/WT-10, MYB62:GFP/agb1-2-1, and MYB62:GFP/agb1-2-4 under $10 \mu \mathrm{M} \mathrm{GA}_{3}$ treatment. The data are the average of three independent experiments, and the error bar represents the SE $(n=10)$. a, b c indicate significant differences. Significant differences were analyzed using Duncan's multiple range test $(p<0.05)$. 


\subsection{The Interaction between AGB1 and MYB62 Did Not Affect the Transcriptional Activation of MYB62}

The subcellular localization of MYB62 protein was completed in WT Col-0 Arabidopsis protoplasts. The results showed that green fluorescent protein (GFP) was expressed in the nucleus and cell membrane of the protoplasts transformed with the vector 16318-AGB1GFP (Figure 5A), indicating that the AGB1 protein was located in the nucleus and cell membrane. GFP was expressed in the nucleus of the protoplasts transformed with the vector 16318-MYB62-GFP (Figure 5A), consistent with a previous report on the localization of MYB62 in plant cells [32] (Devaiah, Madhuvanthi, Karthikeyan, \& Raghothama, 2009). These results indicated that AGB1 and MYB62 are co-located in the nucleus.

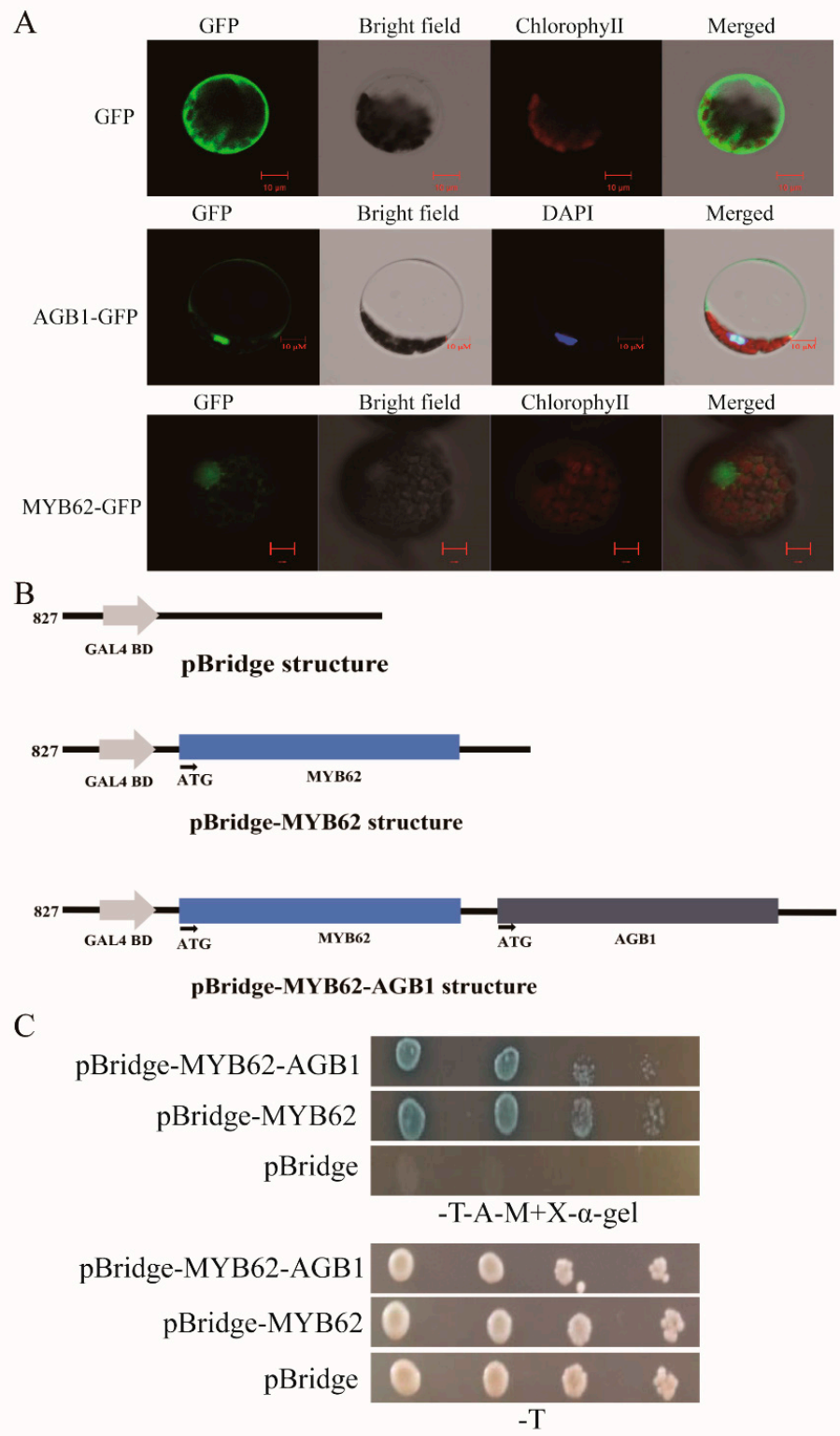

Figure 5. The interaction between AGB1 and MYB62 did not affect the transcriptional activation of MYB62; AGB1 and MYB62 are both located in the nucleus. (A) Analysis of the subcellular localization of AGB1 and MYB62 in the protoplasts of wild-type Arabidopsis Col-0 leaves grown for 3 weeks. Scale bars, $10 \mu \mathrm{m}$. (B) The structure of the pBridge vector, pBridge-MYB62, and pBridge-MYB62-AGB1. (C) The vectors pBridge, pBridge-MYB62, and pBridgeMYB62-AGB1 were transferred into AH109 yeast cells, and the cells were diluted and transferred to selective media (SD/-Trp, SD/-Trp-Ade, $\mathrm{SD} /$-Trp-His-Ade) to observe their growth. 
In order to further explore whether $A G B 1$ affects the transcriptional activity of $M Y B 62$, we carried out transcriptional activation experiments in yeast cells (Figure 5B,C). We constructed a pBridge-MYB62 vector to detect the transcriptional activation activity of MYB62 in yeast cells. In addition, a pBridge-MYB62-AGB1 vector was constructed by inserting AGB1 into pBridge-MYB62, and the effect of $A G B 1$ on MYB62 transcriptional activation was detected (Figure $5 B$ ). The results showed that the yeast transformed with the pBridge-MYB62 vector grew on the selective medium (Figure 5C). The growth of the yeast transformed with pBridge-MYB62-AGB1 was similar to that of pBridge-MYB62 (Figure 5C), indicating that MYB62 had transcriptional activation activity, and $A G B 1$ had no effect on the transcriptional activation of MYB62 in yeast cells.

3.5. MYB62 Can Bind the Promoter of GA Degradation Gene GA2ox7 to Enhance Its Expression, and AGB1 Negatively Regulates the DNA-Binding Activity of MYB62 on the Promoter of GA20x7

$A G B 1$ did not affect the transcriptional activation of $M Y B 62$. Therefore, we speculated that $A G B 1$ may affect the DNA binding activity of MYB62. Thus, we analyzed the expression of many genes related to GA synthesis and degradation in the WT, agb1-2, and MYB62:GFP/WT-10 under $\mathrm{GA}_{3}$ treatment. We found that the GA2ox7 gene (At1g47990), which is related to the degradation process of GA, may be related to the regulation pathway of AGB1-MYB62. Gene expression analysis showed that the expression of GA20x7 in the WT was significantly lower than that in the mutants agb1-2 and N692967, and transgenic plants, including MYB62:GFP/WT-8, MYB62:GFP/WT-10, MYB62:GFP/agb1-2-1, and MYB62:GFP/agb1-2-4 (Figure 6A). This result was consistent with the GA content results (Figure 4F), suggesting that GA20x7 is regulated by AGB1-MYB62. Moreover, we found that the expression of GA2ox7 in MYB62:GFP/agb1-2-1 and MYB62:GFP/agb1-2-4 was similar to that in MYB62:GFP/WT-8 and MYB62:GFP/WT-10, but lower than in the mutants agb1-2 and N692967 (Figure 6A), also consistent with the GA content results (Figure 4F). In addition, EMSA analysis and LUC (luciferase analysis) showed that MYB62 bound to the MYB element (TGGTTG) in the GA2ox7 promoter and enhanced the expression of GA20x7 (Figure 6B-F). However, AGB1 can negatively regulate the binding of MYB62 to the GA20x7 promoter, thus negatively regulating the expression of the GA20x7 promoter in plants (Figure 6G-I). These results indicated that AGB1 interacted with the DNA-binding region of MYB62, further negatively regulating MYB62 binding on the downstream gene $G A 20 x 7$, and actively participating in the GA pathway. 
A

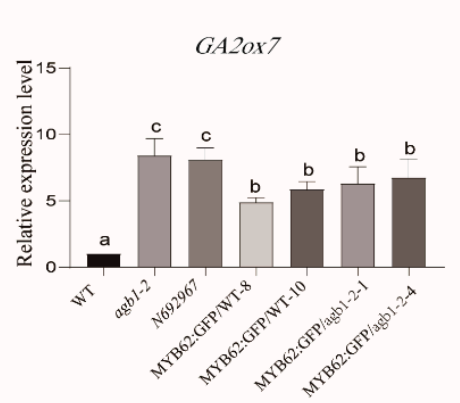

D

Lane $\quad 1 \quad 2 \quad \begin{array}{llll}3 & 4 & 5\end{array}$

MYB62-MBP

MBP

Competatior

Mutation probe

proGA20x7 probe

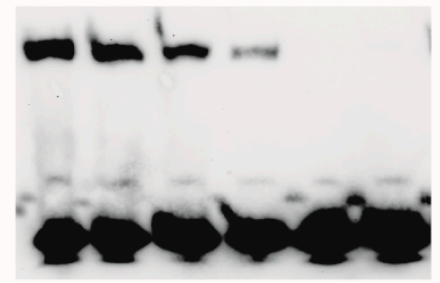

G

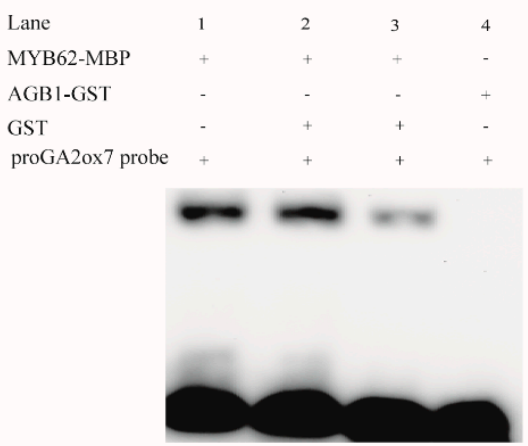

B

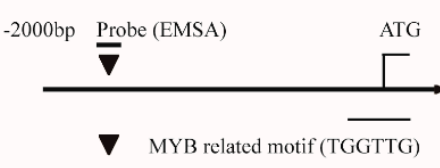

E

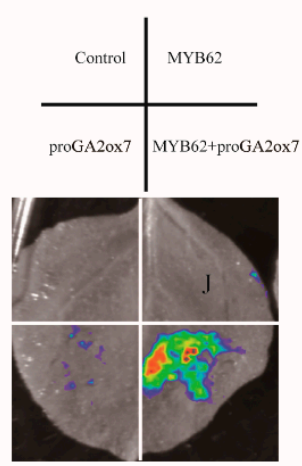

$\mathrm{H}$

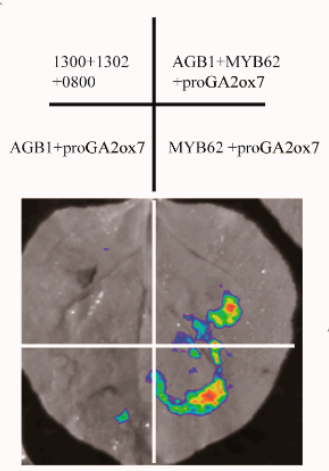

C

Effector

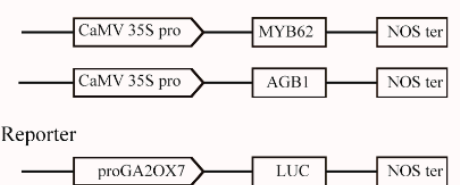

F

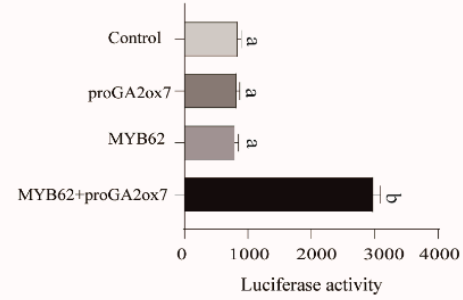

I

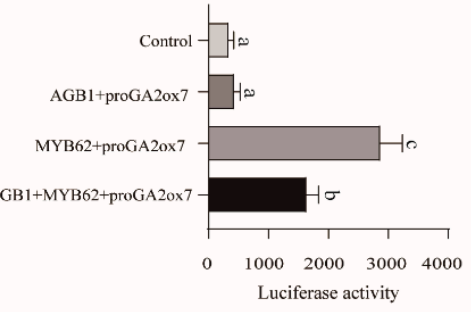

Figure 6. $A G B 1$ negatively regulates the expression of the downstream genes of $M Y B 62$. (A) The GA degradation-related gene, GA2ox7, in the wild-type (Col-0), agb-1-2, N692967, MYB62:GFP/WT-8, MYB62:GFP/WT-10, MYB62:GFP/agb1-2-1, and MYB62:GFP/agb1-2-4 revealed by qRT-PCR analysis under $\mathrm{GA}_{3}$ treatment. Error bars represent the means $\pm \mathrm{SE}(n=3)$. Significant differences were analyzed using Duncan's multiple range test $(p<0.05)$. Relative quantitative results were calculated by normalization using the control gene ACT2 (AT3G18780). (B) Illustration of the MYB62 promoter region showing the presence of the MYB-binding site. (C) Schematic diagram of the effectors and the reporter structure of LUC (luciferase analysis) in E and H. (D) The EMSA (electrophoretic mobility shift assay) experiment showed that MYB62 could bind to the promoter of GA2ox7. (E) The LUC experiment showed that MYB62 could bind to the GA2ox7 promoter and promote the expression of GA2ox7; 302 indicates the pCambia 1302 vector, and 0800 indicates the pGreenII 0800-LUC vector. Representative images of $N$. benthamiana leaves were taken $48 \mathrm{~h}$ after infiltration. All experiments were repeated three times with similar results. (F) Quantification of luminescence intensity in E. Error bars represent the means $\pm \mathrm{SE}(n=3$ ). Significant differences were analyzed using Duncan's multiple range test $(p<0.05)$. (G) The EMSA experiment showed that $A G B 1$ negatively regulates the binding of $M Y B 62$ to the eGA2ox7 promoter. (H) The LUC experiment showed that $A G B 1$ could negatively regulate the binding of $M Y B 62$ to the GA20x7 promoter and negative regulately the expression of GA2ox7; 1300 indicates the pCambia 1300 vector, 1302 indicates the pCambia 1302 vector, and 0800 indicates the pGreenII 0800-LUC vector. Representative images of $N$. benthamiana leaves were taken $48 \mathrm{~h}$ after infiltration. All experiments were repeated three times with similar results. (I) Quantification of luminescence intensity in $\mathrm{H}$. Error bars represent the means $\pm \mathrm{SE}$ $(n=3)$. Significant differences were analyzed using Duncan's multiple range test $(p<0.05)$. 


\section{Discussion}

\subsection{AGB1 Regulates the GA Pathway by Negatively Regulating MYB62 Activity}

In this study, we demonstrated the molecular mechanism of how $A G B 1$ regulates the GA pathway (Figure 7). We found that $A G B 1$ was upregulated and MYB62 was downregulated under $\mathrm{GA}_{3}$ treatment (Figure 3B,C). Both $A G B 1$ and $M Y B 62$ were mainly expressed in the stem of Arabidopsis (Figure 3D,E). Through phenotype analysis of the mutant $a g b 1$ and $M Y B 62$-overexpressing plants under $\mathrm{GA}_{3}$ treatment, $A G B 1$ was found to positively regulate the response to the GA pathway, and $M Y B 62$ negatively regulated the response to the GA pathway (Figure 4). Genetic analysis showed that AGB1 and MYB62 were involved in the same GA pathway, and that MYB62 was downstream (Figure 4). Biochemical experiments showed that AGB1 interacted with the DNA-binding region of MYB62, and that MYB62 could directly bind to the promoter of the GA degradation gene GA2ox7 and promote its expression (Figures 3 and 6). AGB1 inhibited the binding of MYB62 on the GA2ox7 promoter, thus negatively regulating the activity of MYB62 and positively regulating the GA pathway in Arabidopsis (Figure 6G-I).
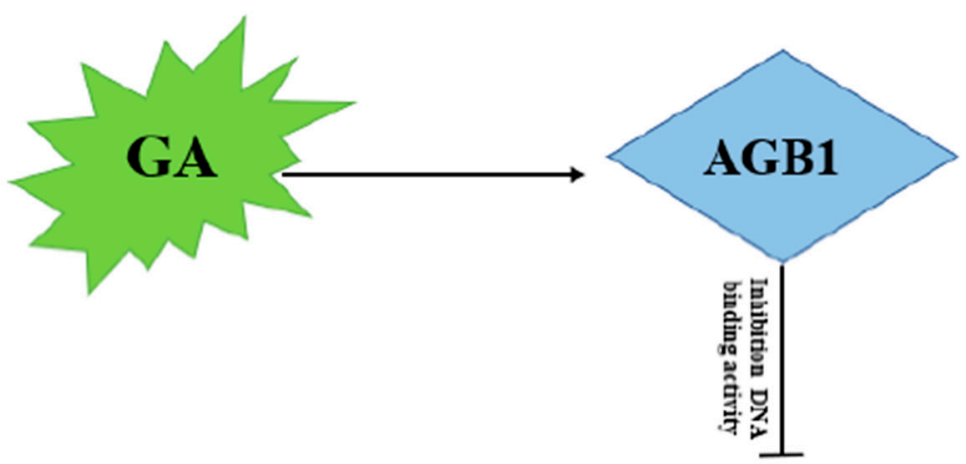

MYB62

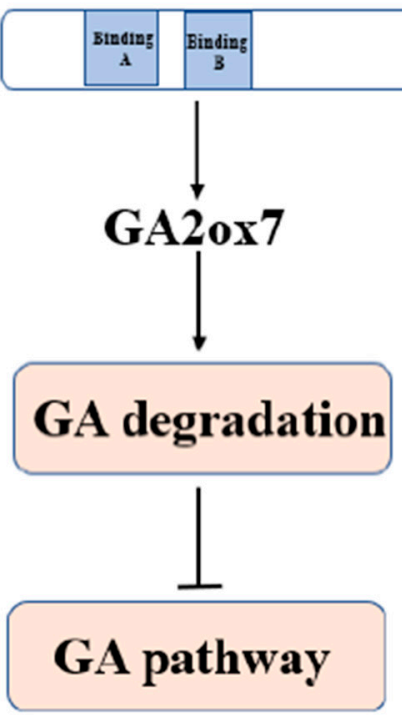

Figure 7. Model of how AGB1 regulates GA-related signaling pathways by controlling MYB62. This regulatory model shows the role of AGB1-MYB62 in regulation of the GA signaling pathway in Arabidopsis thaliana. AGB1 positively participates in the GA signaling pathway, interacts with the negative transcription factor MYB62, inhibits the binding of MYB62 to the promoter of GA metabolism related gene $G A 20 x 7$, and negative regulates $G A 20 x 7$ expression.

We found that the interaction between AGB1 and MYB62 did not affect the transcriptional activation of $M Y B 62$ (Figure 5C). AGB1 interacted with the DNA-binding region of MYB62 (Figure 3A) and negatively regulated the activity of MYB62 by affecting binding to 
the promoter region of the metabolic gene GA20x7 (Figure 6G-I), though neither affected the transcription activity of MYB62. Our previous research on AGB1 in Arabidopsis determined that $A G B 1$ inhibits the transcriptional activation activity of $B B X 21$, a positive regulatory factor, by binding to the transcriptional activation region of $B B X 21$, thereby regulating the expression of the downstream genes [17] (Xu et al., 2017). Taken together, these results suggest that $A G B 1$ regulates the activity of downstream transcription factors by combining different regions of the downstream transcription factors. Other research has found that the G protein $\beta$ subunit regulates plant hypocotyl elongation [17] (Xu et al., 2017), BR signal transduction [37] (Zhang et al., 2018), and plant nutrition regulation [38] (Wu et al., 2020) by interacting with different transcription factors. These results suggest that $A G B 1$ binding with different downstream transcription factors may be a general mechanism to regulate different signaling pathways in Arabidopsis.

In Arabidopsis, the G protein $\alpha$ subunit GPA1 has an important role in regulating hypocotyl elongation, ABA inhibition of the stomatal opening, stomatal density, pollen tube development, and plant height, but the most common regulation mode is the GPA1-GCR1 coupling complex [39] (Chakraborty, Singh, Kaur, \& Raghuram, 2015). In rice, the G$\alpha$ subunit positively regulates the GA pathway by inhibiting the activity of a negative regulator, SLR (slender rice), in GA signaling [13] (Ueguchi-Tanaka et al., 2000). These studies suggested that different subunits of the $G$ protein complex can positively regulate the GA pathway through different downstream genes in plants.

\subsection{The AGB1-MYB62 Pair Is Involved in Regulating the Phosphate Starvation Response in Plants}

MYB62 participates in the response to low-phosphorus stress by negatively regulating the synthesis of GA [32] (Devaiah, Madhuvanthi, Karthikeyan, \& Raghothama, 2009). Therefore, in order to study whether AGB1-MYB62 also functions in phosphorus deficiency, we analyzed the phenotypes of the WT, the agb1-2 mutant, MYB62-GFP/WT-10, and MYB62GFP/agb1-2-4 under phosphorus-free conditions (Supplementary Figure S11). The results showed that under normal conditions, the length of the main root and the number of lateral roots of MYB62:GFP/WT-10 were lower than those of the WT (Supplementary Figure S11). This is consistent with the results of Devaiah et al. (2009). In this study, under normal conditions, there was no significant difference in main root length and lateral root number between $a g b 1-2$ and the WT. Under phosphorus-free conditions, the main root length and lateral root number of agb1-2 were significantly lower than those of the WT, while those of MYB62-GFP/agb1-2-4 were significantly lower than those of the WT and consistent with MYB62-GFP/WT-10 (Supplementary Figure S11). These results indicate that $A G B 1$ positively regulated the response to phosphorus starvation in plants by stimulating root growth. The AGB1-MYB62 pair is also involved in regulating the root growth process under phosphorus starvation. More evidence is needed to demonstrate how the AGB1-MYB62 pair coordinates the regulation of GA and low-phosphorus stress responses in plants.

Supplementary Materials: The following are available online at https:/ /www.mdpi.com/article/10 $.3390 /$ ijms $22158270 / \mathrm{s} 1$. Figure S1 Verification of mutants and transgenic plants. Figure S2 Phenotypes of wild type (Col-0) and agb1-2 under $10 \mu \mathrm{M}$ GA3. Figure S3 Phenotypes of wild type, agb1-2, and N692967 under $1 \mu \mathrm{M}$ GA3 treatment at 1d, 7d, and 10d. Figure S4 Phenotypes of wild type, agb1-2, and N692967 under $10 \mu \mathrm{M}$ GA3 treatment at 1d, 7d, and 10d. Figure S5 Phenotypes of wild type, agb1-2, and N692967 under $100 \mu \mathrm{M}$ GA3 treatment at 1d, 7d, and 10d. Figure S6 Phenotypes of wild type, agb-1-2, N692967, MYB62:GFP/WT-8, MYB62:GFP/WT-10, MYB62:GFP/agb1-2-1, and MYB62:GFP/agb1-2-4 under $1 \mu \mathrm{M} \mathrm{GA3}$ treatment at 1d, 7d, and 10d. Figure S7 Phenotypes of wild type, agb-1-2, N692967, MYB62:GFP/WT-8, MYB62:GFP/WT-10, MYB62:GFP/agb1-2-1, and MYB62:GFP/agb1-2-4 under $10 \mu \mathrm{M}$ GA3 treatment at 1d, 7d, and 10d. Figure S8 Phenotypes of wild type, agb-1-2, N692967, MYB62:GFP/WT-8, MYB62:GFP/WT-10, MYB62:GFP/agb1-2-1, and MYB62:GFP/agb1-2-4 under $100 \mu \mathrm{M}$ GA3 treatment at 1d, 7d, and 10d. Figure S9 Phenotypic identification of wild type, agb-1-2, N692967, MYB62:GFP/WT-8, MYB62:GFP/WT-10, MYB62:GFP/agb1-2-1 
and MYB62:GFP/agb1-2-4 under $1 \mu \mathrm{M}$ GA3 treatment at 10d. Figure S10 Phenotypic identification of wild type, agb-1-2, N692967, MYB62:GFP/WT-8, MYB62:GFP/WT-10, MYB62:GFP/agb12-1, and MYB62:GFP/agb1-2-4 under $100 \mu \mathrm{M}$ GA3 treatment at 10d. Figure S11 Phenotypes of wild type, agb-1-2, N692967, MYB62:GFP/WT-8, MYB62:GFP/WT-10, MYB62:GFP/agb1-2-1, and MYB62:GFP/agb1-2-4 under normal conditions and phosphate-free conditions. Table S1 Primers used in this study. Table S2 Composition of MS powder. Table S3 Composition of MS powder phosphate-free.

Author Contributions: X.Q. and W.T. designed and performed experiments and wrote the manuscript; W.L. contributed to the implementation of the study; Z.H. and W.X. contributed valuable discussions; Z.F., Y.Z., C.W., Z.X., J.C., S.G. and Z.X. provided instructions for the experiments; M.C. coordinated the project, conceived and designed experiments, and edited the manuscript; Y.M. coordinated the project and edited the manuscript. All authors have read and agreed to the published version of the manuscript.

Funding: Key Projects of Genetic Modification (2018ZX08009-17B) and the National Key Project for Research on Transgenic Biology (2016ZX08002-002).

Acknowledgments: This work was supported by the Key Projects of Genetic Modification (2018ZX0800917B), the National Key Project for Research on Transgenic Biology (2016ZX08002-002), and the Agricultural Science and Technology Innovation Program (ASTIP, Transgenic Technology and Application of Crops, and Development and Application of Molecular Markers in Crops).

Conflicts of Interest: The authors declare no conflict of interest.

\section{References}

1. Assmann, S.M. G proteins go green: A plant G protein signaling FAQ sheet. Science 2005, 310, 71-73. [CrossRef]

2. Chen, J.G. Heterotrimeric G-proteins in plant development. Front. Biosci. 2007, 13, 3321. [CrossRef]

3. Assmann, S.M. Heterotrimeric and unconventional GTP binding proteins in plant cell signaling. Plant Cell 2002, 14 (Suppl. S1), S355-S373. [CrossRef]

4. Anantharaman, V.; Abhiman, S.; de Souza, R.F.; Aravind, L. Comparative genomics uncovers novel structural and functional features of the heterotrimeric GTPase signaling system. Gene 2011, 475, 63-78. [CrossRef]

5. Ullah, H.; Chen, J.G.; Wang, S.C.; Jones, A.M. Role of a Heterotrimeric G Protein in Regulation of Arabidopsis Seed Germination. Plant Physiol. 2004, 129, 897-907. [CrossRef]

6. Ullah, H.; Chen, J.G.; Temple, B.; Boyes, D.C.; Alonso, J.M.; Davis, K.R.; Jones, A.M. The b-subunit of the Arabidopsis G protein negatively regulates auxin-induced cell division and affects multiple developmental processes. Plant Cell 2003, 15, 393-409. [CrossRef] [PubMed]

7. Assmann, S.M.; Yu, Y.Q. The heterotrimeric g-protein beta subunit, agb1, plays multiple roles in the Arabidopsis salinity response. Plant Cell Environ. 2015, 38, 2143-2156. [CrossRef]

8. Wang, R.S.; Pandey, S.; Li, S.; Gookin, T.E.; Zhao, Z.X.; Albert, R.; Assmann, S.M. Common and unique elements of the ABA-regulated transcriptome of arabidopsis guard cells. BMC Genom. 2011, 12, 216. [CrossRef] [PubMed]

9. Li, S.J.; Liu, Y.J.; Zheng, L.Y.; Chen, L.L.; Li, N.; Corke, F.; Bevan, M.W. The plant-specific G protein $\gamma$ subunit AGG3 influences organ size and shape in arabidopsis thaliana. New Phytol. 2012, 194, 690-703. [CrossRef]

10. Chen, J.G.; Pandey, S.; Huang, J.; Alonso, J.M.; Ecker, J.R.; Assmann, S.M.; Jones, A.M. GCR1 can act independently of heterotrimeric G-protein in response to brassinosteroids and gibberellins in Arabidopsis seed germination. Plant Physiol. 2004, 135, 907-915. [CrossRef] [PubMed]

11. Pandey, S.; Che, J.G.; Jones, A.M.; Assmann, S.M. G-protein complex mutants are hypersensitive to abscisic acid regulation of germination and postgermination development. Plant Physiol. 2006, 141, 243-256. [CrossRef]

12. Urano, D.; Jones, A.M. Heterotrimeric G protein-coupled signaling in plants. Annu. Rev. Plant Biol. 2014, 65, 365-384. [CrossRef]

13. Ueguchi-Tanaka, M.; Fujisaw, Y.; Kobayashi, M.; Ashikari, M.; Iwasaki, Y.; Kitano, H.; Matsuoka, M. Rice dwarf mutant d1, which is defective in the alpha subunit of the heterotrimeric g protein, affects gibberellin signal transduction. Proc. Natl. Acad. Sci. USA 2000, 97, 11638-11643. [CrossRef]

14. Trusov, Y.; Rookes, J.E.; Tilbrook, K.; Chakravorty, D.; Mason, M.G.; Anderson, D.; Botella, J.R. Heterotrimeric G protein $\gamma$ subunits provide functional selectivity in G $\beta \gamma$ dimer signaling in arabidopsis. Plant Cell 2007, 19, 1235-1250. [CrossRef]

15. Lease, K.A.; Wen, J.Q.; Li, J.; Doke, J.T.; Liscum, E.; Walker, J.C. A mutant Arabidopsis heterotrimeric G-protein beta subunit affects leaf, flower, and fruit development. Plant Cell 2001, 13, 2631-2641. [CrossRef] [PubMed]

16. Xu, D.B.; Chen, M.; Ma, Y.N.; Xu, Z.S.; Li, L.C.; Chen, Y.F.; Ma, Y.Z. A G-Protein $\beta$ Subunit, AGB1, negatively regulates the ABA response and drought tolerance by down-regulating AtMPK6-related pathway in Arabidopsis. PLoS ONE 2015, 10, e0116385. [CrossRef] [PubMed]

17. Xu, D.B.; Ma, Y.N.; Gao, S.Q.; Wang, X.T.; Feng, L.; Li, L.C.; Ma, Y.Z. The G-protein $\beta$ subunit AGB1 promotes hypocotyl elongation through inhibiting transcription activation function of BBX21 in Arabidopsis. Mol. Plant 2017, 10, 1206-1223. [CrossRef] 
18. Utsunomiya, Y.; Samejima, C.; Takayanagi, Y.; Izawa, Y.; Yoshida, T.; Sawada, Y.; Iwasaki, Y. Suppression of the rice heterotrimeric G protein beta-subunit, RGB1, causes dwarfism and browning of internodes and lamina joint regions. Plant J. 2011, 67, 907-916. [CrossRef] [PubMed]

19. Urano, D.; Miura, K.; Wu, Q.Y.; Iwasaki, Y.; Jackson, D.; Jones, A.M. Plant Morphology of Heterotrimeric G protein Mutants. Plant Cell Physiol. 2016, 57, 437-445. [CrossRef] [PubMed]

20. Singh, D.P.; Jermakow, A.M.; Swain, S.M. Gibberellins are required for seed development and pollen tube growth in Arabidopsis. Plant Cell 2002, 14, 3133-3147. [CrossRef]

21. Lange, B.M.; Ghassemian, M. Genome organization in Arabidopsis thaliana: A survey for genes involved in isoprenoid and chlorophyll metabolism. Plant Mol. Biol. 2003, 51, 925-948. [CrossRef]

22. Morrone, D.; Chambers, J.; Lowry, L.; Kim, G.; Anterola, A.; Bender, K.; Peters, R.J. Gibberellin biosynthesis in bacteria: Separate ent-copalyl diphosphate and ent-kaurene synthases in bradyrhizobium japonicum. FEBS Lett. 2009, 583, 475-480. [CrossRef]

23. Davidson, S.E.; Elliott, R.C.; Helliwell, C.A.; Poole, A.T.; Reid, J.B. The pea gene NA encodes ent-kaurenoic acid oxidase. Plant Physiol. 2003, 131, 335-344. [CrossRef]

24. Sakamoto, T.; Miyura, K.; Itoh, H.; Tatsumi, T.; Ueguchi-Tanaka, M.; Ishiyama, K.; Miyao, A. An overview of gibberellin metabolism enzyme genes and their related mutants in rice. Plant Physiol. 2004, 134, 1642-1653. [CrossRef]

25. Hedde, P.; Thomas, S.G. Gibberellin biosynthesis and its regulation. Biochem. J. 2012, 444, 11-25. [CrossRef]

26. Ashikari, M.; Sasaki, A.; Ueguchi-Tanaka, M.; Itoh, H.; Nishimura, A.; Swapan Datta, S.; Khush, S.G. Loss-of-function of a rice gibberellin biosynthetic gene, GA20 oxidase (GA20ox), led to the rice 'green revolution'. Breed. Sci. 2002, 52, 143-150. [CrossRef]

27. Helliwell, C.A.; Chandler, P.M.; Poole, A.; Dennis, E.S.; Peacock, W.J. The CYP88A cytochrome P450, ent-kaurenoic acid oxidase, catalyzes three steps of the gibberellin biosynthesis pathway. Proc. Natl. Acad. Sci. USA 2001, 98, 2065-2070. [CrossRef]

28. Fambrini, M.; Mariotti, L.; Parlanti, S.; Picciarelli, P.; Salvini, M.; Ceccarelli, N.; Pugliesi, C. The extreme dwarf phenotype of the GA-sensitive mutant of sunflower, dwarf2, is generated by a deletion in the ent-kaurenoic acid oxidase1 (HaKAO1) gene sequence. Plant Mol. Biol. 2011, 75, 431-450. [CrossRef] [PubMed]

29. Ueguchi-Tanaka, M.; Ashikari, M.; Nakajima, M.; Itoh, H.; Katoh, E.; Kobayashi, M.; Chow, T.Y.; Matsuoka, M. Gibberellin insensitive dwarf1 encodes a soluble receptor for gibberellin. Nature 2005, 437, 693-698. [CrossRef]

30. Murase, K.; Hirano, Y.; Sun, T.P.; Hakoshima, T. Gibberellin-induced DELLA recognition by the gibberellin receptor gid1. Nature 2008, 456, 459-463. [CrossRef] [PubMed]

31. Shimada, A.; Ueguchi-Tanaka, M.; Nakatsu, T.; Nakajima, M.; Naoe, Y.; Ohmiya, H.; Matsuoka, M. Structural basis for gibberellin recognition by its receptor DID1. Nature 2008, 456, 520-523. [CrossRef] [PubMed]

32. Devaiah, B.N.; Madhuvanthi, R.; Karthikeyan, A.S.; Raghothama, K.G. Phosphate starvation responses and Gibberellic Acid biosynthesis are regulated by the MYB62 transcription factor in Arabidopsis. Mol. Plant 2009, 2, 43-58. [CrossRef] [PubMed]

33. Clough, S.J.; Bent, A.F. Floral dip: A simplified method for Agrobacterium-mediated transformation of Arabidopsis thaliana. Plant J 2010, 16, 735-743. [CrossRef]

34. Livak, K.J.; Schmittgen, T.D. Analysis of relative gene expression data using real-time quantitative PCR and the $2^{-\Delta \Delta C T}$ method. Methods 2001, 25, 402-408. [CrossRef] [PubMed]

35. Yoo, D.S.; Cho, Y.H.; Sheen, J. Arabidopsi mesophyll protoplasts: A versatile cell system for transient gene expression analysis. Nat. Protoc. 2007, 2, 1565-1572. [CrossRef]

36. Yamaji, N.; Huang, C.F.; Nagao, S.; Yano, M.; Sato, Y.; Nagamura, Y.; Ma, J.F. A zinc finger transcription factor ART1 regulates multiple genes implicated in aluminum tolerance in rice. Plant Cell 2009, 21, 3339-3349. [CrossRef]

37. Zhang, T.; Xu, P.B.; Wang, W.X.; Wang, S.; Caruana, J.C.; Yang, H.Q.; Hongli Lian, H.L. Arabidopsis g-protein $\beta$ subunit agb1 interacts with BES1 to regulate brassinosteroid signaling and cell elongation. Front. Plant Sci. 2018, 8, 2225. [CrossRef]

38. Wu, T.Y.; Krishnamoorthi, S.; Goh, H.; Leong, R.; Sanson, A.C.; Urano, D. Crosstalk between heterotrimeric G protein-coupled signaling pathways and WRKY transcription factors modulating plant responses to suboptimal micronutrient conditions. J. Exp. Bot. 2020, 71, 3227-3239. [CrossRef]

39. Chakraborty, N.; Singh, N.; Kaur, K.; Raghuram, N. G-protein signaling components GCR1 and GPA1 mediate responses to multiple abiotic stresses in arabidopsis. Front. Plant Sci. 2015, 6, 1000. [CrossRef] 